\title{
Probiotic and Peanut OIT leads to long-lasting sustained unresponsiveness and quality-of-life improvement in peanut-allergic children
}

\author{
Paxton Loke ${ }^{1}$, Kuang-Chih Hsiao ${ }^{1}$, Adriana Lozinsky ${ }^{1}$, Sarah Ashley ${ }^{1}$, Melanie Lloyd ${ }^{1}$, \\ Sigrid Pitkin ${ }^{1}$, Christine Axelrad ${ }^{1}$, Kaushala Jayawardana ${ }^{1}$, Dean Tey ${ }^{1}$, Ee-Lyn $\mathrm{Su}^{1}$, \\ Marnie Robinson ${ }^{1}$, Agnes Sze Yin Leung ${ }^{1}$, Audrey DunnGalvin² ${ }^{2}$ and Mimi Tang ${ }^{1}$ \\ ${ }^{1}$ Murdoch Childrens Research Institute \\ ${ }^{2}$ University College Cork
}

July 31,2021

\begin{abstract}
Background: Combined treatment with probiotic and peanut oral immunotherapy (PPOIT) was shown to induce sustained unresponsiveness (SU) in a proof-of-concept randomized trial. Additional data on safety and long-term outcomes are needed. This study aimed to evaluate the safety and long-term effects of PPOIT in children with peanut allergy. Methods: Open-label study of 20 children aged 1-12 years with challenge-confirmed peanut allergy; all children received 18-months of PPOIT. Efficacy endpoints were desensitization, 8-week SU, and persistence of 8-week SU at 3-years post-treatment, assessed by double-blind placebo-controlled food challenge (cumulative 4950mg peanut protein). Treatment emergent adverse events and relationship to study treatment were recorded. Immunologic measures and health related quality of life (HRQL) were evaluated at screening, end-of-treatment and 3-years post-treatment. Results: Sixteen children (75\%) completed treatment. By intention-to-treat analysis, $75 \%(15 / 20)$ achieved desensitization and 60\% (12/20) achieved 8-week SU. Ten of 12 participants with SU at endof-treatment consented to the 3-year SU challenge; $6(60 \%)$ had persistence of SU. PPOIT was associated with significantly reduced peanut skin prick test wheal size and serum peanut specific-IgE levels at end-of-treatment, 12-months and 3-years post-treatment. There were no serious adverse events. HRQL scores improved (exceeding the Minimal Clinically Important Difference of 0.45$)$ at 12-months post-treatment with benefit sustained at 3-years post-treatment. Conclusions: Eighteen months of PPOIT induced high rates of desensitization and SU, and SU persisted to 3-years post-treatment in a majority of initial responders. PPOIT led to long-lasting suppression of peanut sIgE and long-lasting clinically important improvement in HRQL.
\end{abstract}

\section{Introduction}

Peanut allergy persists for life in the majority of patients ${ }^{1,2}$. Current management relies on strict allergen avoidance; however, up to $50 \%$ of peanut allergic patients have accidental exposures within 1 year ${ }^{3}$. Moreover, allergic reactions to peanut can be severe, and account for $30 \%$ of food anaphylaxis deaths ${ }^{4}$. There is an unmet need for a treatment that induces long-lasting remission of peanut allergy and improves health-related quality of life (HRQL).

In clinical trials, patient-reported outcomes, such as HRQL, are vital to understand the patient perspective and experience since they capture perceived benefits of treatment not captured by other endpoints. Regulatory agencies increasingly require these data as part of their evaluation process ${ }^{5}$. In drug trials, HRQL is generally only assessed at baseline and at treatment completion; yet assessment of HRQL beyond the treatment phase is needed to ascertain whether benefits are sustained over a longer time period ${ }^{6}$. 
Peanut OIT is effective at inducing desensitization and can induce sustained unresponsiveness (SU) in a subset of treated patients, however there is a paucity of data on long-term effectiveness ${ }^{7,8}$ and impact on HRQL. Few studies have evaluated for long-term SU following peanut OIT; these suggest that OIT-induced SU may be short-lived, with up to $67 \%$ of treatment responders losing their SU status within 12 -months ${ }^{7,8}$. A meta-analysis of peanut OIT studies found that peanut OIT was associated with frequent adverse events and did not lead to significant improvement in $\mathrm{HRQL}^{9}$. Further research to identify novel approaches that improve the efficacy and safety of OIT are needed.

A combined probiotic and peanut oral immunotherapy (PPOIT) treatment was shown to induce desensitization $(83.9 \%)$ and 2-6 week SU $(74.2 \%)$ after 18-months of treatment in a Phase 2a randomized control trial (PPOIT-001 study; ACTRN 12608000594325) ${ }^{10}$. Long-term follow-up of patients showed that PPOITinduced SU persisted to 4 -years post-treatment in $70 \%$ of initial treatment responders ${ }^{11}$. PPOIT treatment also resulted in improved HRQL compared with placebo, with improvement specifically related to the attainment of $\mathrm{SU}^{12}$. Weaknesses of the Phase 2a study included the selection of subjects with peanut allergy based upon a clinical history of reaction and positive peanut skin prick test (SPT) or specific-IgE (sIgE) rather than double-blind placebo-controlled food challenge (DBPCFC) and assessment of SU at 2-6 weeks post-treatment rather than a longer period after treatment.

This study aimed to confirm previous findings using a more stringent 8-week post-treatment SU test in children aged 1-12 years with challenge-confirmed peanut allergy. Safety, HRQL and long-term outcomes were also evaluated.

\section{METHODS}

\section{Study design and eligibility}

This was an open-label, single-arm study of PPOIT for 18-months in 20 children aged 1-12 years with peanut allergy confirmed by failed DBPCFC (up to cumulative 4950mg peanut protein) and positive peanut SPT ([?]3mm) or peanut sIgE ([?]0.35 kUA/L). Pre-determined DBPCFC stopping criteria were applied (supporting information). This study was approved by the Royal Children's Hospital Human Research and Ethics Committee (reference number 35207). Prospective registration was with Australian New Zealand Clinical Trials Registry (ACTRN12615001275550). All participants provided informed consent.

\section{Intervention}

On Day 1, participants received increasing doses of peanut OIT (12\% defatted peanut flour, $50 \%$ peanut protein; Golden Peanut Company) every 20 -minutes to reach a final dose of $12 \mathrm{mg}$ peanut protein (24mg cumulative). A single daily dose of probiotic $\left(2 \times 10^{10}\right.$ cfu Lactobacillus rhamnosus GG ATCC 53103) was also administered. During the subsequent buildup phase, the daily treatment dose was increased every 2weeks until a maintenance OIT dose of $2000 \mathrm{mg}$ peanut protein was reached. Participants continued on a daily maintenance dose of $2000 \mathrm{mg}$ peanut protein until completion of 18-months of PPOIT.

\section{Outcome measures}

\section{Efficacy endpoints}

A DBPCFC (cumulative $4950 \mathrm{mg}$ peanut protein) was performed at the end-of-treatment to assess for desensitization (T2), and at 8-weeks post-treatment (T3) with strict peanut elimination between T2 and T3 to assess for SU. Subjects who achieved SU at T3 were evaluated for persistence of 8-week SU at 3-years post-treatment (T5) by DBPCFC (cumulative 4950mg peanut protein, performed after 8 -weeks of peanut elimination). An optional DBPCFC was offered at 12-months post-treatment (T4) to assess for persistence of SU to 12-months post-treatment in those subjects who achieved SU at T3.

Participants who passed the T2 DBPCFC were classified as desensitized. Participants who passed both the T2 and T3 DBPCFCs were classified as having attained SU and were instructed to include peanut in their dietad libitum. Participants who were desensitized but failed to achieve SU and participants who failed the 
T2 DBPCFC resumed strict peanut avoidance. Participants with SU at T3 who passed the T5 DBPCFC were classified as having persistent SU at 3-years post-treatment.

\section{Treatment emergent adverse events (TEAE)}

A TEAE was any untoward medical occurrence following commencement of treatment, which did not necessarily have a relationship with the study treatment. TEAE were graded according to criteria developed by National Institute of Health (NIH) Consortium for Food Allergy Research (CoFAR) and the Food and Drug Administration (FDA, USA) (supporting information). TEAE are summarized by relationship to treatment and severity, and presented according to medical dictionary for regulatory activities (MedDRA) terms (system organ class and preferred term), and tabulated with descriptive statistics.

\section{Immunological assessments}

SPT (Quintips, standardized peanut extracts and controls; Stallergenes-Greer) and serum/plasma levels of sIgE and sIgG4 to peanut and peanut component (Ara h1, h2, h3, h8 and h9) (ImmunoCAP; Phadia AB, Uppsala, Sweden) were assessed at T0, T2, T3 and T5 timepoints.

\section{Food allergy quality of life questionnaire (FAQLQ)}

HRQL assessments were conducted at screening (T0), T2, T4 and T5. The validated Food Allergy Quality of Life Questionnaire-Parent Form (FAQLQ-PF) was completed by parent/caregiver on behalf of the patient ${ }^{13}$. FAQLQ-PF total score (global impact) and subscales (general emotional impact, food anxiety, social and dietary limitations) were calculated. Lower scores indicate better HRQL (i.e. lower food allergy burden). The minimal clinically important difference (MCID) for FAQLQ-PF is 0.45 points $^{13}$. The food allergy independent measure (FAIM) was used to assess the parent's perception of the chance of an adverse outcome for their child with food allergy ${ }^{14}$.

\section{Statistical methods}

For between timepoint statistical comparisons, parametric variables (SPT wheal size, HRQL) were analyzed using paired t-test with data summarized as mean and standard deviation (SD). Non-parametric variables (sIgE, sIgG4) were analyzed using Wilcoxon rank-sum test with data summarized as median and interquartile range (IQR) and standard t-test on logarithmic scaled values with data summarized as geometric mean and 95\% confidence interval (95\% CI). The number and percentage of participants who achieved desensitization and SU were presented for all enrolled participants (intention-to-treat) and those who had an end-of-treatment DBPCFC outcome (complete case population). Persistence of SU was expressed as the proportion of participants with SU at T3 who maintained SU at 3-years post-treatment (T5). For HRQL outcomes, primary analysis used all available data at all time points. Robustness of findings and impact of missingness was investigated with a sensitivity analysis of complete series data to T4. $\mathrm{P}<0.05$ was statistically significant. Statistical analyses were performed using Stata release 15 software (StataCorp, College Station, Tex) and $\mathrm{R}$ ( $\mathrm{R}$ version 3.6.1).

\section{RESULTS}

\section{Participants}

Twenty children with challenge-confirmed peanut allergy, mean age of 8.2 (SD, 3.0) years were enrolled (Table 1). Four children withdrew during treatment (additional details in supporting information); 16 completed 18-months of treatment and attended the end-of-treatment (T2) and 8-week post-treatment (T3) DBPCFCs; 14 completed T4 and/or T5 DBPCFC (Figure 1).

\section{Efficacy outcomes}

$75 \%(15 / 20)$ and $60 \%(12 / 20)$ of participants achieved desensitization and 8-week SU respectively. Amongst those who completed treatment and had a DBPCFC outcome, 94\% (15/16) achieved desensitization and $75 \%$ $(12 / 16)$ achieved SU. Of the 12 participants with SU at the end-of-treatment (T3), 10 underwent DBPCFC at 3-years post-treatment (T5) following an 8-week period of peanut elimination; 6 had persistent SU. Of 
the 2 participants with SU at T3 who did not undergo the T5 DBPCFC, 1 was lost to follow-up and 1 failed an optional peanut DBPCFC at T4 (12-months post-treatment) and was ineligible for the 3-year posttreatment DBPCFC. The overall rate of persistent SU amongst initial treatment responders was therefore $54.5 \%(6 / 11)$.

\section{Immunological endpoints}

Peanut SPT wheal size decreased significantly from baseline to end-of-treatment $(\mathrm{P}=0.006)$ and remained significantly decreased at both 8 -weeks post-treatment $(\mathrm{P}=0.002)$ and 3 -years post-treatment $(\mathrm{P}=0.001)$. Similarly, there was significant reduction from baseline in peanut sIgE at end-of-treatment $(\mathrm{P}=0.0007)$ that was sustained at 3 -years post-treatment $(\mathrm{P}=0.002)$. Levels of peanut $\mathrm{IIgG} 4$ were significantly increased from baseline at end-of-treatment $(\mathrm{P}=0.0007)$ and at 8 -weeks post-treatment $(\mathrm{P}=0.001)$ (Table 2).

At 3-years post-treatment, participants with persistent SU had lower peanut SPT wheal size (unadjusted $\mathrm{P}=0.06$, age- and sex- adjusted $\mathrm{P}=0.05$ ) and lower peanut sIgE level (unadjusted $\mathrm{P}=0.06$, age- and sexadjusted $\mathrm{P}=0.04$ ) than subjects without persistent $\mathrm{SU}$ (Table 3). Corresponding reductions in $\operatorname{sIgE}$ and increases in sIgG4 against peanut component (Ara h1, h2, h3) were observed at end-of-treatment and 8weeks post-treatment (Table S3 in supporting information). There were no significant changes from baseline in Ara h8 and Ara h9 sIgE and sIgG4 levels.

\section{Safety}

TEAEs are summarized in Table 4. 17/20 (85\%) participants reported a total of 176 treatment-related AE, representing 8.8 AE per participant. No SAEs were reported. Table 5 presents treatment-related AEs reported by at least $10 \%$ of the study population. The most frequently reported treatment-related AEs were abdominal pain (49 events) and hypersensitivity (64 events). The majority of treatment-related AEs were mild $(93 \%, 164 / 176$ events). Hypersensitivity was the most frequently reported treatment-related AE of at least moderate intensity (10 events), followed by wheezing and abdominal pain ( 1 event each). The number of treatment-related AEs by treatment phase were: rush $3(2 \%)$, buildup $110(63 \%)$ and maintenance 63 (35\%) (Table S4 and S5 in supporting information).

\section{Quality of life}

Mean FAQLQ-PF and FAIM total scores reduced significantly, indicating improved HRQL and lower food allergy burden, between end-of-treatment (T2) and 12-months post-treatment (T4); change in score $(95 \%$ CI, p-value) $0.44(0.17-0.70,0.004)$ and $0.37(0.10-0.65,0.01)$ respectively (Table 6). Improvement in HRQL continued, and exceeded the MCID by 3-years post-treatment (T5). Improvement was seen in each of the FAQLQ-PF sub-scores for emotional impact, food anxiety, and social and dietary limitations (Table S6 in supporting information).

\section{DISCUSSION}

PPOIT induced desensitization in $75 \%$ and 8 -week SU in $60 \%$ of patients, with SU persisting at 3-years posttreatment in $55 \%$ of initial treatment responders. These findings support the previous PPOIT-001 randomized trial $^{10}$ results, confirming that PPOIT is highly effective at inducing SU and that SU persists long-term in a majority of treatment responders. Importantly, the PPOIT-002 study applied a more robust measure of SU at end-of-treatment than in the PPOIT-001 trial, with the DBPCFC performed at 8-weeks post-treatment (rather than 2-6 weeks post-treatment) and with a higher cumulative dose of peanut protein (4950mg vs. 4000mg) administered during DBPCFC. Assessment criteria for persistent SU were similar in both studies and yielded similar results with $54.5 \%$ of treatment responders retaining $\mathrm{SU}$ at 3-years post-treatment in the present study (4950mg peanut protein, 8-weeks elimination) and $70 \%$ of treatment responders retaining SU at 4-years post-treatment in PPOIT-001 (4000mg peanut protein, 8-weeks elimination).

Peanut OIT is associated with frequent reactions despite restrictive measures and dosing rules $^{9}$. Gastrointestinal symptoms in particular are common and eosinophilic esophagitis (EoE) has been reported in $2.7 \%$ to 
$5.3 \%$ of treated patients ${ }^{15-17}$. PPOIT treatment in this study did not result in SAEs or EoE. The majority of participants experienced treatment-related AEs, with the majority of these being mild.

Treatment withdrawals may provide a measure of tolerability. Studies of peanut OIT have consistently reported high withdrawal rates due to treatment-related AEs (12\%-15\%), in particular treatment-related gastrointestinal AEs (6-10\% withdrawals) ${ }^{18-20}$. A large phase-3 study evaluating a $300 \mathrm{mg}$ peanut OIT (Palforzia, AR101), reported that $24.5 \%(102 / 416)$ of subjects in the active treatment group withdrew during the treatment phase, with $11.6 \%$ withdrawing due to $\mathrm{AE}^{19}$. In the present study, there were no treatment discontinuations due to PPOIT-related AEs or PPOIT-related gastrointestinal AEs despite 6-7-fold higher maintenance dose; however the sample size is small. Studies have shown Lactobacillus rhamnosus can inhibit intestinal epithelial cell inflammatory responses, increase gut barrier function through increased mucus production, and increase secretory IgA production ${ }^{21-23}$, which provide a plausible pathway for reduced gastrointestinal symptoms and improved tolerability of OIT.

The mechanisms underpinning desensitization and SU remain elusive. Reduction in allergen-specific IgE reflects modulation of the underlying allergic response to allergen and is associated with successful attainment of $\mathrm{SU}^{18,24}$. Studies suggest that longer duration of OIT has greater ability in reducing allergen-specific $\operatorname{IgE}$ levels ${ }^{18,25}$. In this study, 18-months of PPOIT led to significant reduction in circulating peanut and peanut component sIgE levels, and levels continued to decrease over the 3-years post-treatment, suggesting longlasting redirection of the underlying allergic response.

HRQL reflects patient perceived benefits of treatment that are not necessarily captured by other endpoints. When evaluating food allergy treatments, improvement in patient reported outcomes is relevant to assessment of cost effectiveness, benefit over risk, and clinical meaningfulness of endpoint improvements. A meta-analysis of peanut OIT studies, most of which induced desensitization without SU, found no convincing evidence that peanut OIT can improve HRQL ${ }^{9}$. In our study, HRQL/FAQL (FAQLQ-PF/FAIM) improved significantly by 12 -months post-treatment and improvements were sustained at 3-years post-treatment. The greatest improvement was for the sub-scale assessing food-related anxiety. Our findings here are consistent with previous results from the PPOIT-001 randomized trial and support the notion that SU leads to improved quality of life whereas desensitization may not ${ }^{12}$.

The major limitations of this study were the small sample size and open-label design. Known weaknesses of open-label studies include being more at risk of bias and overestimation of treatment-related AEs. Strengths of this study are the comprehensive assessments that were taken, including clinical efficacy, immunological measures and HRQL, as well as the long-term assessment at 3-years post-treatment. Retention from end-oftreatment to 3-years post-treatment was high at $81.25 \%$ (13/16). Additionally, peanut allergy diagnosis was confirmed by DBPCFC at study entry and a more robust measure of SU (longer duration of elimination and higher cumulative challenge dose) was applied in this study compared to the previous PPOIT-001 study.

To conclude, sustained treatment benefit is a vital factor to consider in any clinical trial. Findings from this single-arm open-label study demonstrated that PPOIT induced desensitization and SU in a high proportion of treated patients, with persistence of SU out to 3-years post-treatment in a majority of initial responders, and sustained HRQL improvements. Persistent SU was associated with lasting reduction in peanut sIgE, providing compelling evidence that PPOIT modulated the underlying allergic response with durable effect. Further studies are needed to clarify whether the addition of probiotic to peanut OIT contributes to efficacy and/or safety outcomes.

\section{Acknowledgments}

We thank the PPOIT-002 study doctors and nurses, RCH Clinical Trials Pharmacy, RCH Immunology Laboratory (technical assistance with the ImmunoCAP assays) and MCRI Biobanking. We thank the children and parents who participated in the PPOIT-002 study. We also thank Metagenics for provision of probiotic and placebo treatments and Abacus-ALS for supplying consumables for sIgE and sIgG4 testing. This work also contributes to the Australian National Health and Medical Research Council's Centre for Food \& Allergy Research. 


\section{Key message}

There is paucity of data on peanut OIT long-term effects and quality of life impact. PPOIT induces sustained unresponsiveness that persists to 3-years post-treatment in a high proportion of treated patients, and leads to long-lasting improvement in health-related quality of life, supporting previous findings from a proofof-concept randomized trial. PPOIT provides lasting benefits for children with peanut allergy, offering an approach to induce long-term sustained unresponsiveness and improve health-related quality of life.

\section{References}

1. Ho MH, Wong WH, Heine RG, et al. Early clinical predictors of remission of peanut allergy in children. $J$ Allergy Clin Immunol . 2008;121 (3):731-6.

2. Skolnick HS, Conover-Walker MK, Koerner CB, et al. The natural history of peanut allergy. J Allergy Clin Immunol . 2001;107 (2):367-74.

3. Bock SA, Atkins FM. The natural history of peanut allergy. J Allergy Clin Immunol . 1989;83:900-4

4. Sampson HA, Mendelson L, Rosen JP. Fatal and near-fatal anaphylactic reactions to food in children and adolescents. N Engl J Med . 1992;327 (6):380-4.

5. Baldwin M, Spong A, Doward L, et al. Patient-reported outcomes, patient-reported information: from randomized controlled trials to the social web and beyond.Patient . 2011;4 (1):11-7.

6. Haslam A, Herrera-Perez D, Gill J, et al. Patient Experience Captured by Quality-of-Life Measurement in Oncology Clinical Trials. JAMA Netw Open . 2020;3 (3):e200363.

7. Chinthrajah RS, Purington N, Andorf S, et al. Sustained outcomes in oral immunotherapy for peanut allergy (POISED study): a large, randomised, double-blind, placebo-controlled, phase 2 study. Lancet . 2019;394 (10207):1437-49.

8. Syed A, Garcia MA, Lyu SC, et al. Peanut oral immunotherapy results in increased antigen-induced regulatory T-cell function and hypomethylation of forkhead box protein 3 (FOXP3). J Allergy Clin Immunol . 2014;133 (2):500-10.

9. Chu DK, Wood RA, French S, et al. Oral immunotherapy for peanut allergy (PACE): a systematic review and meta-analysis of efficacy and safety. Lancet . 2019;393 (10187):2222-32.

10. Tang ML, Ponsonby AL, Orsini F, et al. Administration of a probiotic with peanut oral immunotherapy: A randomized trial. J Allergy Clin Immunol . 2015;135 (3):737-44 e8.

11. Hsiao K-C, Ponsonby A-L, Axelrad C, et al. Long-term clinical and immunological effects of Probiotic and Peanut Oral Immunotherapy (PPOIT) post-treatment cessation: Four-year follow-up of a randomized double-blind placebo-controlled trial.Lancet Child Adolesc Health . 2017;1 (2):97-105.

12. Dunn Galvin A, McMahon S, Ponsonby AL, et al. The longitudinal impact of probiotic and peanut oral immunotherapy on health-related quality of life. Allergy . 2018;73 (3):560-8.

13. DunnGalvin A, de BlokFlokstra BM, Burks AW, et al. Food allergy QoL questionnaire for children aged 0-12 years: content, construct, and cross-cultural validity. Clin Exp Allergy . 2008;38 (6):977-86.

14. Van der Velde JL, Flokstra-de Blok BM, Vlieg-Boerstra BJ, et al. Development, validity and reliability of the food allergy independent measure (FAIM). Allergy . 2010;65 (5):630-5.

15. Echeverría-Zudaire L, Fernández-Fernández S, Rayo-Fernández A, et al. Primary eosinophilic gastrointestinal disorders in children who have received food oral immunotherapy. Allergol Immunopathol . 2016;44 (6):531-6.

16. Lucendo AJ, Arias A, Tenias JM. Relation between eosinophilic esophagitis and oral immunotherapy for food allergy: a systematic review with meta-analysis. Ann Allergy, Asthma Immunol . 2014;113 (6):624-9. 
17. Petroni D, Spergel JM. Eosinophilic esophagitis and symptoms possibly related to eosinophilic esophagitis in oral immunotherapy. Ann Allergy, Asthma Immunol . 2018;120 (3):237-40.

18. Vickery BP, Scurlock AM, Kulis M, et al. Sustained unresponsiveness to peanut in subjects who have completed peanut oral immunotherapy. J Allergy Clin Immunol . 2014;133 (2):468-75.

19. Vickery BP, Vereda A, Casale TB, et al. AR101 Oral Immunotherapy for Peanut Allergy. N Engl J Med. 2018;379 (21):1991-2001.

20. Virkud YV, Burks AW, Steele PH, et al. Novel baseline predictors of adverse events during oral immunotherapy in children with peanut allergy. J Allergy Clin Immunol . 2017;139 (3):882-8.

21. Miettinen M, Matikainen S, Vuopio-Varkila J, et al. Lactobacilli and streptococci induce interleukin-12 (IL-12), IL-18, and gamma interferon production in human peripheral blood mononuclear cells. Infect Immun . 1998;66 (12):6058-62.

22. Pessi T, Sutas Y, Hurme M, et al. Interleukin-10 generation in atopic children following oral Lactobacillus rhamnosus GG. Clin Exp Allergy.2000;30 (12):1804-8.

23. Rautava S, Arvilommi H, Isolauri E. Specific probiotics in enhancing maturation of IgA responses in formula-fed infants. Pediatr Res . 2006;60 (2):221-4.

24. Narisety SD, Frischmeyer-Guerrerio PA, Keet CA, et al. A randomized, double-blind, placebo-controlled pilot study of sublingual versus oral immunotherapy for the treatment of peanut allergy. $J$ Allergy Clin Immunol . 2015;135 (5):1275-82.e1-6.

25. Jones SM, Pons L, Roberts JL, et al. Clinical efficacy and immune regulation with peanut oral immunotherapy. J Allergy Clin Immunol . 2009;124 (2):292-300, .e1-97.

Table 1. Participant Baseline Demographics

\begin{tabular}{|c|c|}
\hline & PPOIT-002 study $(\mathrm{n}=20)$ \\
\hline Age $(y)$ & \\
\hline Median (IQR) & $8.36(6.34,10.83)$ \\
\hline Mean (SD) & $8.2(3.0)$ \\
\hline Male sex & \\
\hline $\begin{array}{l}\text { n }(\%) \\
\text { History of doctor-diagnosed eczema (ever) }\end{array}$ & $14(70)$ \\
\hline $\begin{array}{l}\mathrm{n}(\%) \\
\text { History of doctor-diagnosed asthma (ever) }\end{array}$ & $8(40)$ \\
\hline $\begin{array}{l}\text { n }(\%) \\
\text { Peanut SPT wheal size }(\mathrm{mm})\end{array}$ & $3(15)$ \\
\hline $\begin{array}{l}\text { Mean (SD) } \\
\text { Peanut sIgE }(k U A / L)\end{array}$ & $12.3(4.5)$ \\
\hline Median (IQR) & $69.9(7.9,100.0)$ \\
\hline
\end{tabular}

$\mathrm{IQR}=$ interquartile range, $\mathrm{SD}=$ standard deviation, $\mathrm{SPT}=$ skin prick test

Table 2. Summary of immunological endpoints (peanut SPT, sIgE and sIgG4)

\begin{tabular}{lll}
\hline Peanut SPT Wheal Size $(\mathbf{m m})$ & Mean $(\mathrm{SD}), \mathrm{n}$ & P-value* $^{*}$ \\
\hline Baseline & $12.3(4.5), 20$ & $\mathrm{~N} / \mathrm{A}$ \\
End-of-treatment & $7.0(4.1), 16$ & 0.006 \\
8-weeks post-treatment & $8.1(4.4), 16$ & 0.002
\end{tabular}




\begin{tabular}{lll}
\hline Peanut SPT Wheal Size $(\mathbf{m m})$ & Mean $(\mathrm{SD}), \mathrm{n}$ & P-value* $^{*}$ \\
\hline 3-years post-treatment & $7.3(3.3), 13$ & 0.001 \\
Peanut sIgE (kUA/L) & Median $(\mathrm{IQR}), \mathrm{n}$ & P-value** \\
Baseline & $69.9(7.9,100.0), 20$ & $\mathrm{~N} / \mathrm{A}$ \\
End-of-treatment & $7.4(1.2,33.6), 15$ & 0.0007 \\
8-weeks post-treatment & $10.2(1.0,27.6), 16$ & 0.0004 \\
3-years post-treatment & $2.5(1.0,18.9), 12$ & 0.002 \\
Peanut sIgG4 (mgA/L) & Median $(\mathrm{IQR}), \mathrm{n}$ & $\mathrm{P}$-value** \\
Baseline & $0.5(0.2,1.3), 16$ & $\mathrm{~N} / \mathrm{A}$ \\
End-of-treatment & $21.6(6.0,93.5), 15$ & 0.0007 \\
8-weeks post-treatment & $11.2(3.3,28.4), 14$ & 0.001 \\
3-years post-treatment & $\mathrm{N} / \mathrm{A}$ & $\mathrm{N} / \mathrm{A}$ \\
\hline
\end{tabular}

*Paired t-test with equal variance; ${ }^{* *}$ Wilcoxon signed rank test; $\mathrm{N} / \mathrm{A}=$ not available. $\mathrm{P}$-values are derived from statistical comparisons between each timepoint with baseline.

Table 3. Peanut SPT wheal size and SIgE level of those who had persistent SU and those who did not at 3-years post-treatment

\begin{tabular}{lll}
\hline & Mean $(\mathrm{SD})$ of SPT $(\mathrm{mm}), \mathrm{n}$ & Mean $(\mathrm{SD})$ of SPT $(\mathrm{mm}), \mathrm{n}$ \\
\hline 3-years post-treatment & Persistent SU & No Persistent SU \\
Peanut SPT & $5.6(2.3), 6$ & $9.0(2.7), 4$ \\
& Geometric mean $(95 \% \mathrm{CI})$ of sIgE (kUA/L), n & Geometric mean $(95 \% \mathrm{CI})$ of $\operatorname{sIgE}(\mathrm{kUA} / \mathrm{L}), \mathrm{n}$ \\
3-years post-treatment & Persistent SU & No Persistent SU \\
Peanut sIgE & $0.8(0.2,4.1), 6$ & $11.6(0.3,487.5), 4$ \\
\hline
\end{tabular}

\#Unadjusted; *Age-, sex- adjusted regression; **Age-, sex- adjusted regression (of log-transformed values)

Table 4. Overall Summary of TEAEs

$(\mathrm{N}=20)$

Number of participants with TEAEs

Number of participants with Treatment-Related TEAEs*

Number of participants with Moderate or Severe TEAEs

Number of participants with Treatment-Related*, Moderate or Severe TEAEs

Number of Treatment-Emergent Adverse Events

Number of Treatment-Related* TEAEs

Number of Moderate or Severe TEAEs

Number of Treatment-Related*, Moderate or Severe TEAEs

Number of TEAE per participant

Number of Treatment-Related* TEAEs per participant

Number of Moderate or Severe TEAEs per participant

Number of Treatment-Related*, Moderate or Severe TEAEs per participant

\section{All participants}

$20(100 \%)$

$17(85 \%)$

$9(45 \%)$

$7(35 \%)$

249

176

22

12

12.45

8.8

1.1

0.6

*Treatment-related TEAEs = "Probably Related", "Possibly Related".

Table 5. Treatment-Related Adverse Events Reported by at Least $10 \%$ of the Study Population 
System Organ Class, Preferred Term

$(\mathrm{N}=20)$

$(\mathrm{N}=20)$

\section{Relationship}

All Treatment Related TEAEs

Gastrointestinal Disorders

Abdominal pain

Vomiting

Gastrointestinal disorder

Immune System Disorders

Hypersensitivity

Skin and Subcutaneous Tissue

Disorders

Urticaria

Respiratory, Thoracic and

Mediastinal Disorders

Allergic respiratory symptoms

\section{All Participants \\ All Participants}

Probably
$17(85 \%)[118](5.90)$
$12(60 \%)[57](2.85)$
$7(35 \%)[42](2.10)$
$8(40 \%)[12](0.60)$
$3(15 \%)[3](0.15)$
$11(55 \%)[38](1.90)$
$11(55 \%)[38](1.90)$
$5(25 \%)[15](0.75)$
$5(25 \%)[15](0.75)$
$3(15 \%)[7](0.35)$
$3(15 \%)[4](0.20)$

Possibly

$[58]^{\mathrm{a}}(2.90)$

$1(5 \%)[14](0.70)$

$1(5 \%)[7](0.35)$

$1(5 \%)[7](0.35)$

0

$1(5 \%)[26](1.30)$

1 (5\%) [26] (1.30)

$2(10 \%)[11](0.55)$

$2(10 \%)[11](0.55)$

$1(5 \%)[6](0.30)$

[2] $(0.10)$

TEAEs are presented as $\mathrm{N}(\%)[\mathrm{E}](\mathrm{PP})$ where $\mathrm{N}$ equals the number of subjects to experience that event, (\%) equals the percentage of subjects to experience that event, [E] equals the number of events experienced, and $(\mathrm{PP})$ equals the number of events per participant. If a participant has more than one $\mathrm{AE}$ coded to the same MedDRA term, the participant is counted only once per treatment, for the AE with least favourable relationship.

Table 6. Difference in FAQLQ total scores between timepoints

\begin{tabular}{lllll}
\hline Timepoints of interest & FAQLQ total score (points) & FAQLQ total score (points) & FAQLQ total score (points)* & FAG \\
\hline & Sample size & Mean difference & P-value & $95 \%$ \\
T0-T2 & 16 & 0.32 & 0.25 & $(-0.2 .0$ \\
T0-T4 & 14 & 0.57 & 0.08 & $(-0.0$ \\
T0-T5 & 10 & 0.65 & 0.08 & $\left(0.1^{1}\right.$ \\
T2-T4 & 14 & 0.44 & $\mathbf{0 . 0 0 4}$ & $(0.0$ \\
T2-T5 & 10 & 0.68 & $\mathbf{0 . 0 4}$ & $(-0.3$ \\
T4-T5 & 10 & 0.26 & 0.34 & \\
\hline
\end{tabular}

*FAQLQ total score is a food allergy specific health-related quality of life measure (maximum of 30 questions; each scored 0-6; 0 representing best quality of life).

**FAIM total score assesses the parent's perception of the chance of an adverse outcome for the child with a food allergy (six questions with 6-point response scale; 0 (extremely unlikely) to 6 (extremely likely)).

Figure 1. CONSORT diagram of participant flow in the PPOIT-002 study

\section{Hosted file}

Figure 1 - PPOIT-002 LTSU \& QoL improvement_23062021.docx available at https://authorea.com/ users/428429/articles/532285-probiotic-and-peanut-oit-leads-to-long-lasting-sustainedunresponsiveness-and-quality-of-life-improvement-in-peanut-allergic-children 